\title{
Research on Irregular Field Area Measurements Metamorphosis Testing Method
}

\author{
Bin $\mathrm{Hu}$, Wei Tang ${ }^{*}$, Zhanwei Hui and Ruihao Ding \\ Institute of Command Information System, PLAUST, JIANG Su, NAN Jing, China
}

\begin{abstract}
Aiming at the problem that it difficult to judge the correctness of the testing output during the area measurement testing, this paper purposes applying metamorphosis testing to terrain measurement software which shows its good effect. Taking the metamorphosis testing for irregular field area in terrain measurement software as application background, an improved area measurement is proposed. On the basis of this algorithm, the relation of metamorphosis and the criterion of judgment are constructed, and the experimental verification is carried out by metamorphosis relation for area division.
\end{abstract}

Keywords-programming teaching methods; SPOC; mixed learning

\section{BACKGROUND}

Area measurement is one of the important functions of terrain measurement. Its function is to calculate the area of the selected area on the map. Usually, the area to be calculated is an irregular figure and it's hard for tester to find an existing math formula to get an expected output. If the formula used by the algorithm is applied, the computational workload will increase dramatically, and there are limited use cases can be examined so that the testing adequacy is difficult to guarantee.

Area measurement is used to implement the terrain quantification analyzing function which is hard for manually implementation. This kind of program is belong to the category described as Programs which were written to determine the answer. If the correct answer were known, there would have been no need to write the program by Weyuker ${ }^{[1]}$. Obviously, area measurement program belongs to non-testable programs described by Weyuker, which exist testing decision problem.

\section{METAMORPHOSIS TESTING}

In order to solve the testing decision problem, Chan has proposed a software metamorphosis testing method based on metamorphosis relation ${ }^{[2]}$. By reusing the test cases, the method verifies whether or not the outputs of multiple use cases satisfies the relational attribute that the software program must satisfy, and this type of relation attribute is called metamorphosis relation. Based on the raw data, the testing method take pre-extracted metamorphosis relation as judgement criterion. When the input satisfies certain requirements, the checking module will check whether or not the output satisfy the metamorphosis relation. If it doesn't satisfy the relation, it is said there are some flaws in the software.
Definition: Metamorphosis Relation(MR) refers to the relation satisfied by different inputs and corresponding outputs with different testing programs. That is to say, the attributes that a program must satisfy is called a metamorphosis relation. Metamorphosis relation is the core of execution result of metamorphosis testing, and its quality directly affects the validity of the testing. The relation can be of any necessary one, including but not restricted to identical relation, neither limited to single execution. Sometimes metamorphosis relation may relate to the relationship between multiple execution results. Assume that the program $P$ is used to implement the function $f, x_{1}, x_{2}, \ldots, x_{n}(n>1)$ are different input variables of $f$, the corresponding outputs are $f\left(x_{1}\right), f\left(x_{2}\right), \ldots, f\left(x_{n}\right)$. If the inputs $x_{1}, x_{2}, \ldots, x_{n}$ satisfy the relation $r$ and the outputs $f\left(x_{1}\right), f\left(x_{2}\right), \ldots, f\left(x_{n}\right)$ can be deduced to satisfy the relation $\mathrm{r}_{\mathrm{f}}$, that is:

$$
r\left(x_{1}, x_{2}, \ldots, x_{n}\right) \Rightarrow r_{f}\left(f\left(x_{1}\right), f\left(x_{2}\right), \ldots, f\left(x_{n}\right)\right)
$$

$\left(r, r_{f}\right)$ is called the metamorphosis relation of $P$.

As a result, if program $P$ is correct it must satisfy the derivation $(2-2)$ :

$$
r\left(I_{1}, I_{2}, \ldots, I_{n}\right) \Rightarrow r_{f}\left(P\left(I_{1}\right), P\left(I_{2}\right), \ldots, P\left(I_{n}\right)\right)
$$

If the designed test case does not run up to the upper model, the assumption is incorrect and the program is wrong. The metamorphosis relation is the core of the execution of the metamorphosis test, and its quality directly affects the validity of the testing. For different software tested, the software metamorphosis relation is usually more than one should be satisfy with. If $M R_{i}=\left(r_{i}, r_{f_{i}}\right)$ is the $i$-th metamorphosis relation of program $P$, then $M R=\left(M R_{1}, M R_{2}, \ldots, M R_{n}\right)$ is metamorphosis relation set of $P$. The main process of software metamorphosis testing is shown in Fig. 1. 


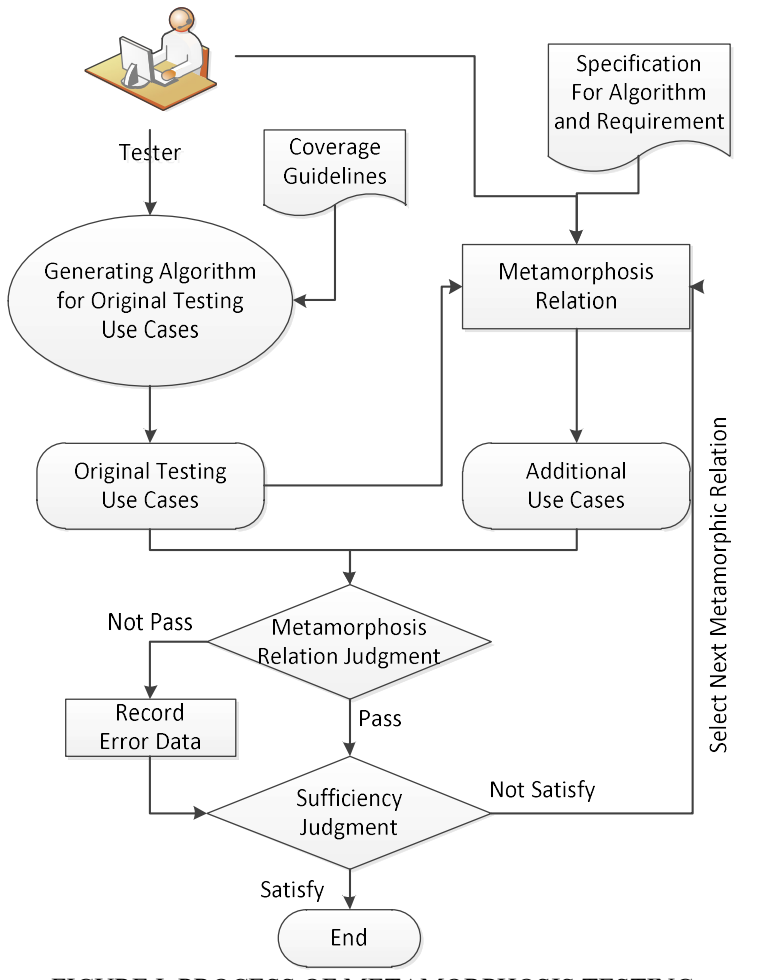

FIGURE I. PROCESS OF METAMORPHOSIS TESTING

\section{Metamorphosis Testing Based IRREgUlar Field Area Measurement Program Testing}

The boundary curves of irregular graphs can be regarded as closed intervals composed of piecewise continuous curves . The boundary curves are not necessarily smooth in a whole, and the more irregular the graph is, the more segments are divided. Finding the area of an irregular region is an integral problem in fact. The mainly area measurements for irregular field include trapezoidal method, Simpson numerical integral method and cubic spline function ${ }^{[4]}$. The trapezoidal method is usually used in the instance which have equal intervals on[a,b] This method only uses the function value to prove the area of the field and it is simple and easy to implement with computer. When the number of segments reaches a certain amount, the result will be much more accurate, so it is a commonly used methods. Although other improved methods are more accurate than the trapezoidal method, the algorithm is complex and difficult to implement, and the improvement of accuracy is limited.

The two forms of trapezoidal method are fixed step method and variable step method. The fixed step method is rather simple but it is hard to determine the reasonable step size in advance. And the variable step method can improve the calculation accuracy by adjusting the step size little by little but it is more complex to implement it. This paper presents a composite approach including the advantages of the two algorithms mentioned above.

Assume that there is a piecewise smooth irregular region $A$ as shown in Fig. 2. The method used in the trapezoidal method is to refine the quadrature interval. In this method, a set of segmentation point are insert between the interval $[a, b]$, making $a<x_{1}<\ldots<x_{n}<b$. And then, calculate the approximate area of sub interval $\left[\mathrm{x}_{\mathrm{k}}, \mathrm{x}_{\mathrm{k}+1}\right]$ using the expression $s_{k}=h\left(f\left(x_{k}\right)+f\left(x_{k+1}\right)\right) / 2$, and get the sum $\mathrm{S}=\sum_{i=1}^{n} S_{i}$. The distance between $x_{k}$ and $x_{k+1}$ is the integral step size and more the size is small more the result is accuracy.

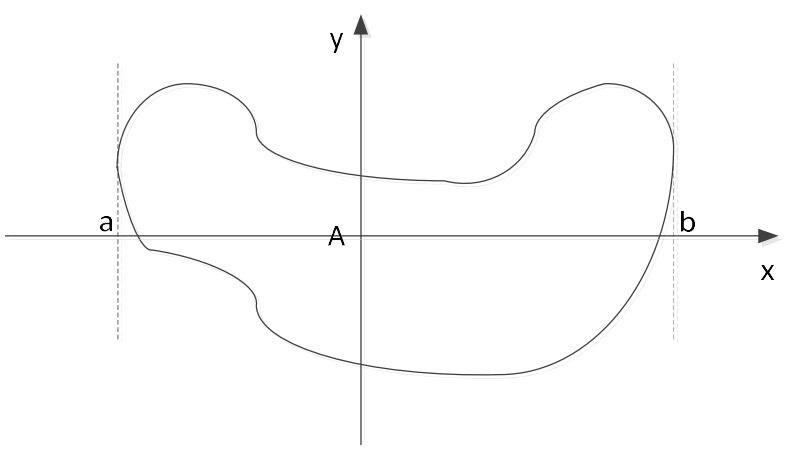

FIGURE II. SMOOTH IRREGULAR REGION EXAMPLE

This paper takes the step of successive binary division principle to divide the interval[a, b] into $2^{n}$ segments and inserts $2^{n}-1$ equidistant points $\mathrm{x}_{1}, \mathrm{x}_{2}, \ldots, \mathrm{x}_{2^{n}-1}$, and set the integral step size $h=\frac{b-a}{2^{n}}$. The area of the field is:

$$
S_{n}=\frac{1}{2} h_{n}\left[f(a)+2 \sum_{i=1}^{2^{n}-1} f\left(x_{i}\right)+f(b)\right]
$$

In this method, it is difficult to determine the proper value of $n$, this paper proposed an algorithm to increase the value of $n$ in a loop to get the field area value of $S_{n}$ and using the judging expression $\left|S_{n}-S_{n-1}\right|<\gamma$ to determine when to exit the loop.

HUANG applied metamorphosis testing to area measurement program for the first time and solved the decision problem of area calculation program testing ${ }^{[5]}$. Nine different metamorphosis relations which can be applied to area measuring are proposed and proved to be functional. According to the characteristics of irregular field measurement this paper selects the area division metamorphosis relation to take the experiments. In the viewpoint of mathematics, the area division metamorphosis relation belongs to linear combination metamorphosis relation. Assume $f$ is the function for irregular field measurement and program $P_{f}$ is used to implement the function with input $I$ and output $P_{f}(I)$. The area division metamorphosis relation is:

$$
\begin{aligned}
& I_{0}=k_{1} I_{1}+k_{2} I_{2}+\ldots+k_{n} I_{n} \Rightarrow f\left(I_{0}\right)=k_{1} f\left(I_{1}\right)+ \\
& k_{2} f\left(I_{2}\right)+\ldots+k_{n} f\left(I_{n}\right)
\end{aligned}
$$

The area division metamorphosis relation is a linear combination metamorphosis with the values of $k_{1}, k_{2}, \ldots, k_{n}$ are 1 .

The irregular region has its particularity relative to the regular polygon. Because the irregular field area measurement depends on continuous dividing, edge fitting and calculating, the result of it is an estimated value which have error compare to the true value, so the error must be taken into concern when judging whether or not the output satisfy the metamorphosis 
relation. Analyzing the calculation accuracy of trapezoid method, obviously, the terrain is a simulation of the actual terrain, and the edge is smooth or piecewise smooth curve.

Theorem: Assume that the function $\mathrm{f}(\mathrm{x})$ is continuous in the closed interval[a, b], and in the open interval $(a, b)$, it's continuous differential at finite points, and there is a positive integer $\mathrm{M}$ which satisfy $\left|f^{\prime}(x)\right| \leq M$. Using a set of dividing point $\mathrm{a}=x_{0}<x_{1}<\ldots<x_{n}=b$ divide the interval $[\mathrm{a}, \mathrm{b}]$ into equal parts, then as following:

$\left|\int_{a}^{b} f(x) d x-\frac{b-a}{2 n}\left[f(a)+f(b)+2 \sum_{i=1}^{n-1} f\left(x_{i}\right)\right]\right| \leq \frac{M(b-a)^{2}}{4 n}(5)$

According to the formulae 3-3, the error of the area calculated by the algorithm mentioned above is:

$$
\begin{aligned}
& \left|S_{n}-\int_{a}^{b} f(x) d x\right| \leq \frac{M(b-a)^{2}}{4 \times 2^{n}} \\
& \left|\int_{a}^{b} f(x) d x-S_{n-1}\right| \leq \frac{M(b-a)^{2}}{4 \times 2^{n-1}}
\end{aligned}
$$

Adding the formula 3-4 and 3-5 will get the formulae 3-6:

$$
\left|S_{n}-\int_{a}^{b} f(x) d x\right|+\left|\int_{a}^{b} f(x) d x-S_{n-1}\right| \leq \frac{M(b-a)^{2}}{4 \times 2^{n}}+\frac{M(b-a)^{2}}{4 \times 2^{n-1}}
$$

$$
\left|S_{n}-S_{n-1}\right| \leq 3 \times \frac{M(b-a)^{2}}{4 \times 2^{n}}
$$

Because $\left|S_{n}-S_{n-1}\right| \leq \gamma$ is used as the termination condition in the algorithm, assume that:

$$
\left|S_{n}-S_{n-1}\right|=\beta
$$

substituting formulae 3-8 into formulae of 3-7 can get the formulae 3-9 as following:

$$
\beta / 3 \leq \frac{M(b-a)^{2}}{4 \times 2^{n}}
$$

Because the trapezoidal method make the value of area $S_{n}$ approaching to the truth value of $\int_{a}^{b} f(x) d x$ the area by increasing the division number $n$, and both $\left|S_{n}-\int_{a}^{b} f(x) d x\right|$ and $\left|S_{n}-S_{n-1}\right|$ are convergent, according to the formula 3-5 and 3-9, when the algorithm finishes, it can be considered that the calculation error is small enough and there is the following formulae:

$$
\left|S_{n}-\int_{a}^{b} f(x) d x\right| \approx \beta / 3
$$

Due to the unknowability of irregular area, the value of the left part of equation (3-10) is unknown. In the following part, the value of $\beta / 3$ is taken to analyze the error. The area measurement accurate to two decimal places. That is to say, $\gamma=0.01, \beta \leq 0.01, \beta / 3 \leq 0.0033$. From the empirical data it is known that when $\beta / 3=0.0033$ then difference between $\beta / 3$ and $\left|S_{n}-\int_{a}^{b} f(x) d x\right|$ is less than 0.0001 .

\section{CASE VERIFICATION}

In order to illustrate the effectiveness of metamorphosis testing technology in detecting errors, some variation is implanted in the program under test which is used to check the ability to find the errors for metamorphosis testing. This method is called mutated analyzing technology ${ }^{[7]}$. In this paper, the most common mutation operators including application arithmetic operator substitution, relation operator substitution and constant substitution are adapted to generate tree typical mutation for tested program. For illustrative purposes, the source code in the PloyArea function is mutated as list in TABLE I.

TABLE I. TABLE STYLES

\begin{tabular}{|c|c|c|}
\hline Mutation & Original code & Code with errors \\
\hline M1 & for $($ int $\mathrm{i}=1 ; \mathrm{i}<\mathrm{I} ; \mathrm{i}++) ;$ & for $($ int $\mathrm{i}=1 ; \mathrm{i}<=\mathrm{I} ; \mathrm{i}++)$ \\
\hline M2 & return $(2 * \text { sum }+\mathrm{f}(\mathrm{a})+\mathrm{f}(\mathrm{b}))^{*} \mathrm{~h} / 2$ & return $(2 * \text { sum }-\mathrm{f}(\mathrm{a})+\mathrm{f}(\mathrm{b}))^{*} \mathrm{~h} / 2$ \\
\hline M3 & return $(2 * \text { sum }+\mathrm{f}(\mathrm{a})+\mathrm{f}(\mathrm{b}))^{*} \mathrm{~h} / 2$ & return $(4 * \text { sum }-\mathrm{f}(\mathrm{a})+\mathrm{f}(\mathrm{b}))^{*} \mathrm{~h} / 2$ \\
\hline
\end{tabular}

This paper select a geographic information system as a test software to be measured, the system can not only measure rectangle, circle, polygon and other areas, but also to calculate the map on the lakes and other natural terrain area. Define linear combination metamorphosis relation as 3-2. According to the analysis in [8] and experiment result in [9], the linear combination metamorphosis relation has strong ability of error detection. As all the outputs of area measurement are positive values, the following changes can be made:

$$
\begin{gathered}
P_{f}\left(I_{0}\right) \pm \varepsilon_{0}=k_{1}\left(P_{f}\left(I_{1}\right) \pm \varepsilon_{1}\right)+k_{2}\left(P_{f}\left(I_{2}\right)\right. \\
\left. \pm \varepsilon_{2}\right)+\ldots k_{n}\left(P_{f}\left(I_{n}\right) \pm \varepsilon_{n}\right) \\
P_{f}\left(I_{0}\right)-k_{1} P_{f}\left(I_{1}\right)-k_{2} P_{f}\left(I_{2}\right)-\cdots-k_{n} P_{f}\left(I_{n}\right) \\
=\mp \varepsilon_{0} \pm k_{1} \varepsilon_{1} \pm k_{2} \varepsilon_{2} \pm \ldots \pm k_{n} \varepsilon_{n}
\end{gathered}
$$

Replacing $\varepsilon_{0}, \varepsilon_{1}, \ldots, \varepsilon_{n}$ with $\beta_{0} / 3, \beta_{1} / 3, \ldots, \beta_{n} / 3$, can get the following result:

Judgment criterion: The absolute value of the relative difference $\mid\left(P_{f}\left(I_{0}\right)-k_{1} P_{f}\left(I_{1}\right)-k_{2} P_{f}\left(I_{2}\right)-\cdots-k_{n} P_{f}\left(I_{n}\right)\right) /$ $P_{f}\left(I_{0}\right) \mid$ identity to $\left|\beta_{0}-k_{1} \beta_{1}-k_{2} \beta_{2}-\cdots-k_{n} \beta_{n} / 3_{f}\left(I_{0}\right)\right|$. Taking into account the deviation caused by the replacement, the calculation error less than $\left(1+k_{1}+k_{2}+\cdots+k_{n}\right) \times$ $10^{-4} / P_{f}\left(I_{0}\right)$ is allowed.

The longitudinal or transverse cutting of the experimental area is divided into two to seven ranges. The test results show that without regarding to the error all the use cases of the tested program do not satisfy the metamorphosis relation of the area division leading to correctness of the subsequent metamorphosis testing can not be guaranteed. The judgment criterion for metamorphosis relation given in this paper can deal with the impact of calculation error on metamorphosis 
relation. The experimental results show that the criterion can guarantee the source program to satisfy the metamorphosis relation and detect the mutation embedded in the program.

\section{CONCLUSIOIN}

According to the analysis and experiment, metamorphosis testing applied to terrain calculation program testing can effectively solve the measurement problem and has a good effect. During the testing process, the selection of the metamorphosis relation calculation error has a crucial impact on the metamorphosis test results. Based on the in-depth analysis of algorithm and error, the experiments have proved that the choice of error threshold can not only guarantee the original program to meet the metamorphosis relationship, but also detect the implanted mutation. It has a strong convincingness and practical significance.

\section{REFERENCES}

[1] E. J. Weyuker. On testing non-testable programs[J]. The Computer Journal, 25(4): 465-470, 1982.

[2] F. T. Chan, T. Y. Chen, I. K. Mak and Y. T. Yu. Proportional Sampling Strategy: Guidelines for Software Testing Practitioners. Information and Software Technology[J], 38(12):775-782, 1996.

[3] Song Huang,Ding Ruihao,Li Hui.Research on metamorphosis testing of slop and aspect calculating progroms[J].Journal of Computre Applications. 33(6):1657-1661, 2013.

[4] GUO Jianfeng, GUI Qingming, ZHU Jianqing,A Numerical Method of computing the Area of Irregular Field[J]. Bulletin of Surveying and Mapping,2001,01(02):12-13

[5] Song Huang, Yiting Duanmu, Zhanwei Hui, Mengyu Ji. Metamorphosis Testing of Spatial Distance Measuring Function of GIS[C]. International Conference on Computer Application and System Modeling (ICCASM). xiamen, China 2011, 671-674.

[6] Andrews JH, Briand LC and Labiche Y: Is mutation an appropriate tool for testing experiments?[C]. In Proceedings of the 27th International Conference on Software Engineering (ICSE05) 2005, 402-411.

[7] Mayer, J., and Guderlei, R., An Empirical Study on the Selection of Good Metamorphosis Relations[C], in Proceedings of the 30th Annual International Computer Software and Applications Conference(COMPSAC'06), Chicago, USA, 2006. 